\title{
LEUKOCYTE COUNT AND TWO-PART DIFFERENTIAL IN WHOLE BLOOD BASED ON A PORTABLE MICROFLOW CYTOMETER
}

\author{
W. Shi ${ }^{1}$, S. Zheng ${ }^{1,2}$, H.L. Kasdan ${ }^{3}$, A. Fridge and Y.C. Tai $^{3}$ \\ ${ }^{1}$ California Institute of Technology, Pasadena, California, USA \\ ${ }^{2}$ Pennsylvania State University, University Park, Pennsylvania, USA \\ ${ }^{3}$ IRIS Diagnostics Division, IRIS International Inc., Chatsworth, California, USA
}

\begin{abstract}
This work demonstrated leukocyte count and two-part leukocyte differential from the whole blood based on a portable microflow cytometer system. Leukocytes are selectively stained with a fluorescent dye, Acridine Orange (AO). The blood sample is then pumped through a disposable microfluidic device for fluorescence sensing. Under blue LED excitation (460nm), the green fluorescence from DNA and the red fluorescence from RNA are detected simultaneously with two photomultiplier tubes (PMT). Leukocytes are counted and differentiated into two parts, lymphocyte versus non-lymphocyte, based on their fluorescence signatures. The results, including leukocyte absolute count and lymphocyte ratio, matched well with the commercial hemacytometer counts (maximal error 9.0\%, correlation coefficient $\sim 0.8$ ). We also tested the system for the first time under a zero-gravity (zero-G) environment that facilitated its application in space missions.
\end{abstract}

\section{KEYWORDS}

Leukocyte, Count, Differential, Fluorescence, Acridine Orange

\section{INTRODUCTION}

Leukocyte count with differential is one of the most common clinical tests performed nowadays. It provides important diagnostic information for diseases such as viral/bacterial infections, inflammations, allergies and the leukemia [1]. Besides, once the therapy is started, the test helps to monitor patient's response to various treatments and recovery from illness. Leukocyte count also finds useful applications in the space mission. For instance, the lymphocyte ratio among leukocytes serves as a biodosimetry indicator of astronaut's exposure to space radiation.

Currently the test is performed either manually on an optical microscope or on an automated hemacytometer. The manual count takes a trained physician to distinguish each cell by observation, and the result is not very accurate. The automated count has been proven to be more accurate and time efficient. However, the conventional automated hemacytometers are usually bulky and expensive. A micro blood counter holds the promise of a point-of-care solution with less cost and smaller footprint.

Particle sensing (beads, erythrocyte or cultured cell lines) have been demonstrated on microfluidic devices by detecting the electrical impedance [2], scattered light [3] and fluorescent emission [4]. Previously we presented a feasibility study of leukocyte count with fluorescent sensing on an optical bench configuration [5]. In this work, we demonstrated the leukocyte count and two-part differential (lymphocyte versus non-lymphocyte) from the whole blood based on an improved portable system. We also validated the system under a zero-G environment for human space missions.

\section{DETECTION PRINCIPLE}

Acridine orange is a cell permeable dye which mainly stains the nucleic acid. We use AO to selectively stain leukocytes in the whole blood obtained with fingerprick or venipuncture [6]. The DNA in leukocytes stains green (emission peak wavelength $525 \mathrm{~nm}$ ) and the RNA stains red (emission peak wavelength $650 \mathrm{~nm}$ ). Mature erythrocytes in human blood lack DNA and RNA. Therefore normal erythrocytes are not stained by AO and don't interfere with stained leukocytes. Leukocytes are then counted by detecting the green and red fluorescence signals from individual leukocyte in a microfluidic channel. Leukocytes two part differential, i.e. lymphocyte vs. non-lymphocyte, is achieved based on their different fluorescence signatures [7]. The specific staining of leukocytes by AO eliminates the need of erythrocytes lysis.

Conventional automated hemacytometers use hydrodynamic focusing with sheath flow to guide samples into a small region of the detection zone. A large volume of buffer fluid is necessary even for a small volume of the testing sample. In our device, the use of sheath flow is avoided by constraining cells within the physical dimension of the microfluidic channel. Therefore the total fluid volume, which limits the on-chip integration of the whole system, is largely reduced comparing to commercial hemacytometers.

\section{DEVICE FABRICATION}

The device was fabricated with standard soft lithography [5]. Briefly, a silicon wafer mold was made with lithography and deep reactive ion etching (DRIE). Then the polydimethylsiloxane (PDMS) prepolymer was mixed and casted onto the mold. After a brief baking, the PDMS sheet was peeled off and the channel pattern on the mold was transferred to the PDMS block. The device was finished by bonding the PDMS block to a glass substrate. 
Fig.1 shows the microfluidic channel on a fabricated device. The channel depth is $16 \mu \mathrm{m}$ and the channel width of the detection zone is $28 \mu \mathrm{m}$. The thickness of the PDMS block is controlled to be less than $6 \mathrm{~mm}$ in order to accommodate the working distance of the optical condensing lens. PDMS and glass are chosen as the device material for the ease of fabrication and their excellent optical properties in the wavelength range of this study.

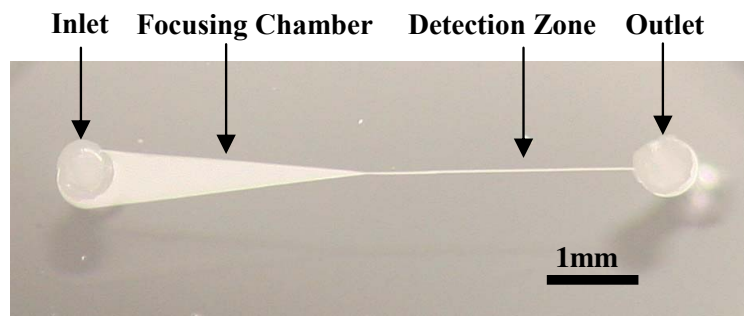

Figure 1: Top view of the fabricated microfluidic device. The channel depth was 16 1 m. The width of the detection zone was $28 \mu \mathrm{m}$. The thickness of the whole device is less than $6 \mathrm{~mm}$.

\section{PORTABLE SYSTEM CONFIGURATION}

A portable prototype was constructed with commercial off-shelf components. As shown in Fig.2, the optical components were assembled inside a black housing to be shielded away from the environmental light. The microfluidic chip was mounted into the cage and could be replaced easily. The whole system, including all the electrical components and the data acquisition module, was enclosed within an aluminum case of 12" x 9" x 5" in volume. It was powered by either the $110 \mathrm{~V}$ AC or a $5 \mathrm{~V} \mathrm{DC}$ source, and the recorded data was read out by a laptop through a USB port.

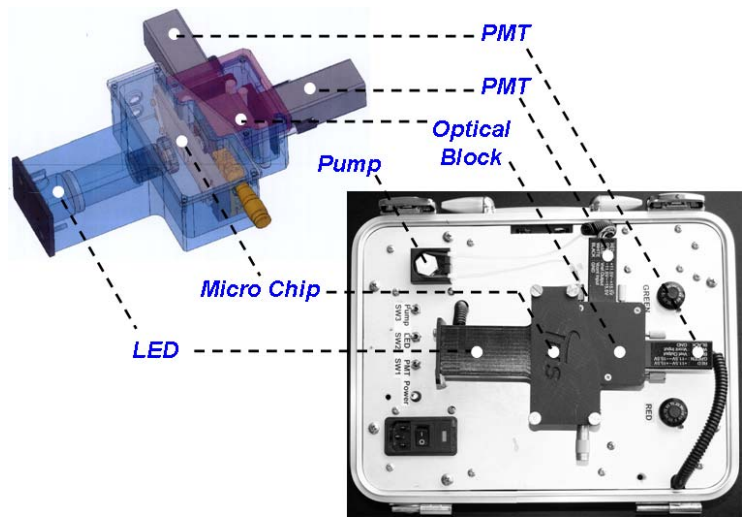

Figure 2: The portable microflow cytometer prototype for leukocyte count and differential. The upper-left schematic showed the design of the optical housing. The lower-right picture showed the whole system except the laptop for signal visualization and data storage.

The optical configuration is illustrated in Fig.3. A blue LED with $460 \mathrm{~nm}$ center wavelength is utilized as the excitation source. The emitting light is collected with a narrow slit, purified by an excitation filter and condensed to the detection zone. With a uniform illumination on the microfluidic channel, the hydrodynamic focusing with sheath flow is not required. Fluorescent emission from the detection zone is collected and split into a green $(510 \mathrm{~nm}-560 \mathrm{~nm})$ channel and a red $(>590 \mathrm{~nm})$ channel by one dichroic mirror and two emission filters inside the optical block. Two PMTs are adopted to measure the intensity of the fluorescent emission, which is converted into digital signals by the data acquisition module. The stained sample is loaded conveniently either by leaving a sample-loaded pipette tip connected to the inlet port or by inserting the inlet-tubing directly into a vertical sample vial. A mini peristaltic pump is used to draw the sample through the microflow cytometer.

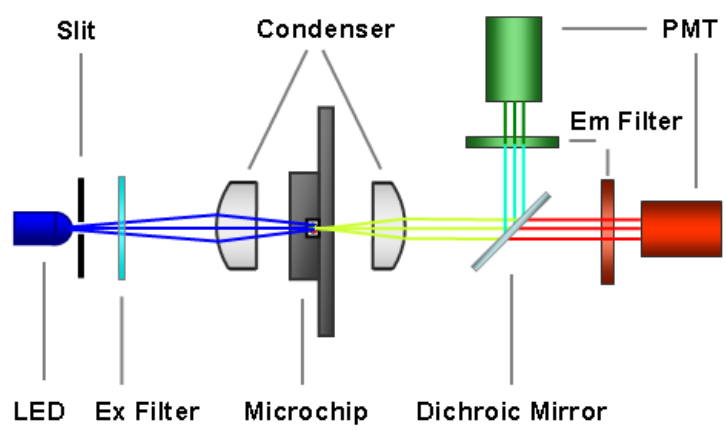

Figure 3: Schematic view of the optical configuration.

\section{RESULT AND DISCUSSION}

Fresh human whole blood with EDTA anticoagulant was purchased from HemaCare Corporation, CA, and tested on the microflow cytometer prototype. A known volume of blood, e.g. $5 \mu \mathrm{L}$, was drawn with a volumetric micropipette. The blood sample was stained with $100 \mu \mathrm{g} / \mathrm{ml}$ $\mathrm{AO}$ for 2 minute at room temperature, and then diluted ten times with an aqueous solvent Ficoll-Paque before loading. Ficoll-Paque slows down the blood cell sedimentation process, thus can extend testing time. The total sample volume was $50 \mu \mathrm{L}$ or less. The pump rate could be adjusted from $1.0 \mu \mathrm{L} / \mathrm{min}$ to $6.0 \mu \mathrm{L} / \mathrm{min}$. A sampling rate of $50 \mathrm{kHz}$ was used for data acquisition.

Detected fluorescence signals were stored and visualized on a laptop. Fig. 4 shows the typical time traces of the detected signals. Each peak represents a stained leukocyte flowing through the detection channel. Leukocytes are detected for their green fluorescence from DNA and red fluorescence from RNA simultaneously. The signal-to-noise-ratio (SNR) is 12 for the green peaks and 18 for the red peaks on average. The red signal has a higher SNR because the emission peak wavelength of AO shifts from $520 \mathrm{~nm}$ (Green) to $650 \mathrm{~nm}$ (Red) when binding to RNA, which helps to distinguish the stained leukocytes from the green background. 


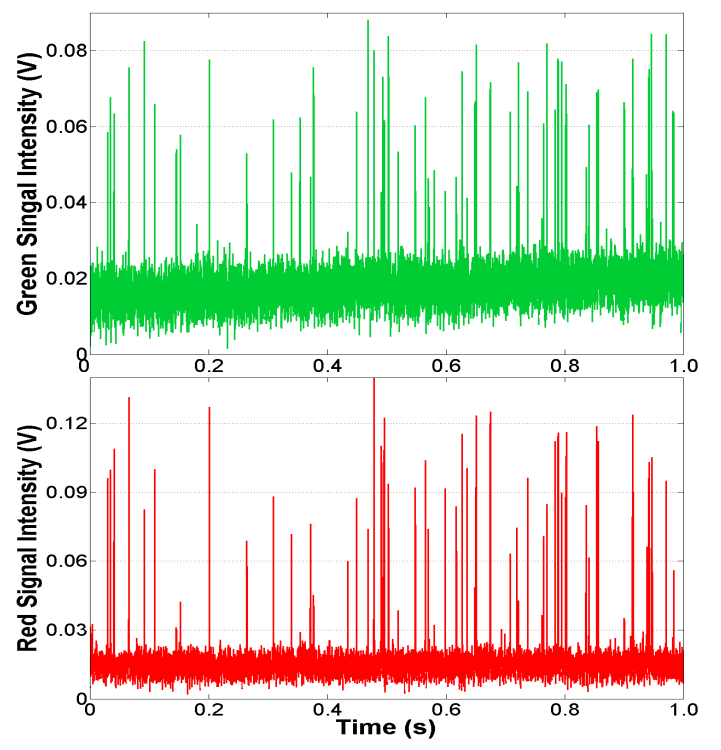

Figure 4: Time traces of measured signals from both the green channel and the red channel simultaneously. The blood sample was stained with $100 \mu \mathrm{g} / \mathrm{ml} \mathrm{AO}$.

Fig.5 is the scatter plot with the green fluorescence intensity versus the red fluorescence intensity detected from leukocytes. The plot shows two clusters clearly separated. The upper-left cluster is generated by lymphocytes, whereas the lower-right cluster is contributed by granulocytes and monocytes. Three-cluster differentiation is possible by $\mathrm{AO}$ staining with a low staining temperature and short testing time [7]. In our previous report, leukocyte was differentiated with only the red fluorescence signal, as shown in the inset of Fig.5. The two-parameter signature from the current system shows better discrimination of lymphocytes against non-lymphocytes, thus improves the accuracy of the differential.

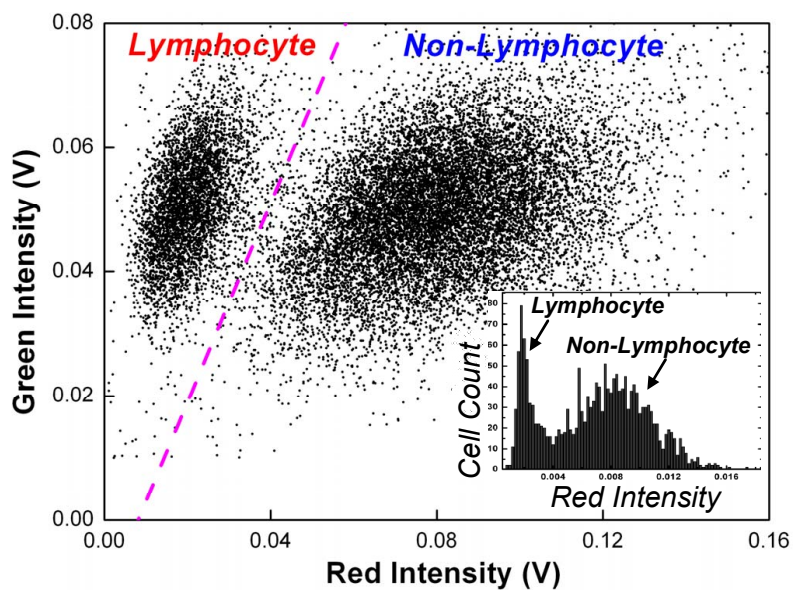

Figure 5: Scatter plot of green fluorescence intensity vs. red fluorescence intensity detected from leukocytes. The inset plot shows leukocyte differentiation by only red fluorescence in our previous report.
Fig.6 compares the results of leukocyte absolute count and lymphocyte ratio between our microflow cytometer and a commercial hemacytometer. The absolute count was calculated as dividing the detected leukocyte events by the volume of blood known from sample preparation. The lymphocyte ratio was calculated as the percentage of the counted lymphocyte events within the total leukocyte events. For five trials with different blood samples, the results match those from commercial hemacytometer counts by a maximal error $9.0 \%$ and a correlation coefficient $\sim 0.8$, which is within the error range of the commercial hemacytometer and the error range for clinical significance.

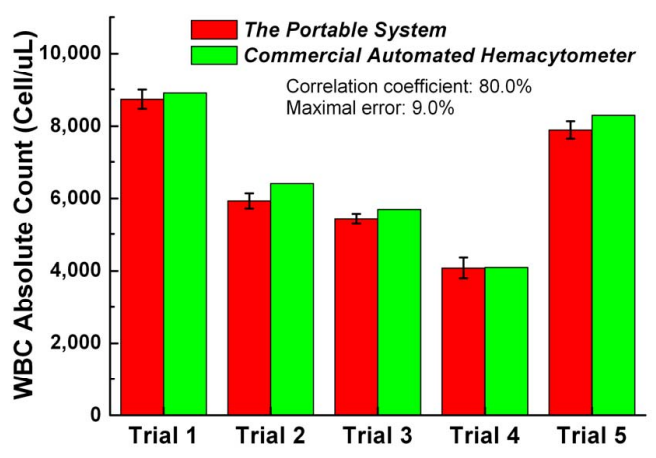

(a)

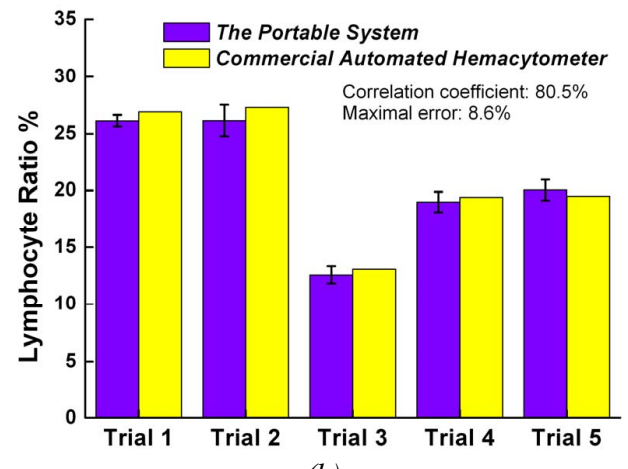

(b)

Figure 6: Results of (a) leukocyte absolute count, and (b) lymphocyte ratio, compared with counts from a commercial hemacytometer. Error bar shows the standard deviation of 3 runs in each trial.

Recently, we tested the prototype system on a zero-G flight at NASA Johnson Space Center. The system underwent a transition between zero-G and two-G periodically during the test as the airplane repeated a parabolic trajectory. The testing blood sample was prepared by the same staining recipe as the on ground test and loaded on the prototype system before takeoff. The system was initiated to run the sample and to record signals before the airplane started the parabolic trajectory. The acceleration data in terms of G-force was recorded by a separate accelerometer on the airplane. 


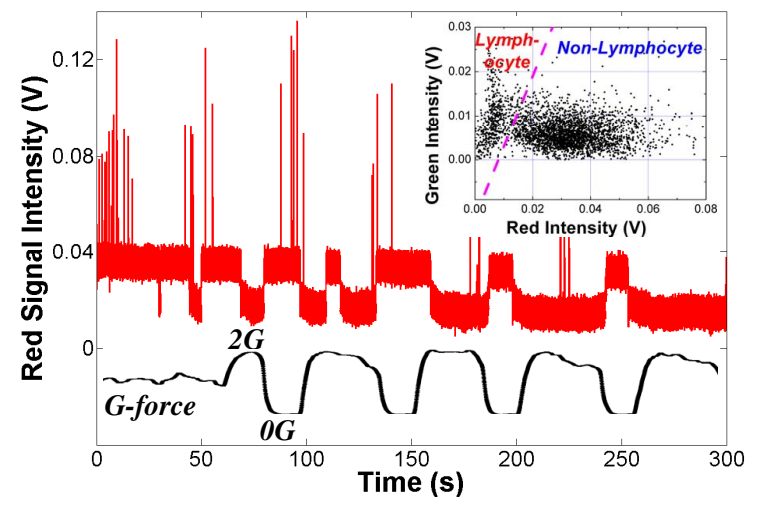

Figure 6: Part of the fluorescent signals detected during the zero-G flight test. The inset shows the obtained scatter plot of the total data for two-part differential. The bottom curve shows the measured real-time G force during the test.

As shown in Fig.7, the detected fluorescent signals from leukocytes shows no observable difference from those of the ground tests, and the similar two-cluster pattern is obtained in the scatter plot. This result is reasonable as the gravity has no direct impact on the optical fluorescence and has only negligible influence on the cells' position inside the microfluidic channel, which is dominated by the flow dynamics. The zero-G and two-G cycles can be visualized due to the baseline shift of the signal. We believe this is caused by the slight displacement of the microfluidic device and can be avoided by fastening the device more tightly in the future.

\section{CONCLUSION}

We fabricated a microfluidic device and constructed a portable system to demonstrate leukocyte count and two-part differential from human whole blood. Leukocytes were selectively stained by $100 \mu \mathrm{g} / \mathrm{ml} \mathrm{AO}$ and diluted ten times with Ficoll-Paque. Leukocyte was counted and differentiated into two parts, lymphocyte versus non-lymphocyte, by the green $(510 \mathrm{~nm}-560 \mathrm{~nm})$ and red $(>590 \mathrm{~nm})$ fluorescence signature. Results of leukocyte absolute count and lymphocyte ratio matched the commercial hemacytometer counts with a maximal error $9.0 \%$ and a correlation coefficient $\sim 0.8$. The prototype was also validated on a zero-G flight test.

\section{ACKNOWLEDGEMENTS}

This work was supported by NSBRI/NASA under the Award Number TD01301. The authors especially thank Shannon Melton and Victor Hurst IV from Wyle Integrated Science and Engineering for their help on zero-G flight test. The authors also wish to thank all members of Caltech Micromachining Group for helpful discussions.

\section{REFERENCES}

[1] B. Houwen, "The Differential Cell Count", Laboratory
Hematology, vol. 7, pp. 89-100, 2001.

[2] S. Gawad, L. Schild and P. Renaud, "Micromachined Impedance Spectroscopy Flow Cytometer for Cell Analysis and Particle Sizing", Lab on a chip, vol. 1, pp. 76, 2001.

[3] Z. Wang, J. El-Ali, M. Engelund, T. Gotsad, I.R. Perch-Nielsen, K.B. Mogensen, D. Snakenborg, J.P. Kutter and A. Wolff, "Measurements of Scattered Light on a Microchip Flow Cytometer with Integrated Polymer Based Optical Elements", Lab on a chip, vol. 4, pp. 372-377, 2004.

[4] T.D. Perroud, J.N. Kaiser, J.C. Sy, T.W. Lane, C.S. Branda, A.K. Singh and K.D. Patel, "Microfluidic-Based Cell Sorting of Francisella Tularensis Infected Macrophages Using Optical Forces", Anal. Chem., vol. 80, pp. 6365-6372, 2008.

[5] S. Zheng, J.C.H. Lin, H.L. Kasdan, and Y.C. Tai, "Fluorescent Labeling, Sensing and Differentiation of Leukocytes from Undiluted Whole Blood Samples", Sensors and Actuators B, vol. 132, pp. 558-567, 2008.

[6] L.R. Adams and L.A. Kamentsky, "Machine Characterization of Human Leukocyte by Acridine Orange Fluorescence", Acta Cytologica, vol. 15, pp. 289-291, 1971.

[7] J.A. Steinkamp, A. Romero and M.A. Van Dilla, "Multiparameter Cell Sorting: Identification of Human Leukocytes by Acridine Orange Fluorescence", Acta Cytologica, vol. 17, pp. 113-117, 1973.

\section{CONTACT}

*Y.C. Tai, tel: +1-626-395-8317; yctai@mems.caltech.edu 